\title{
A Solanum lycopersicoides reference genome facilitates biological discovery in tomato
}

Adrian F. Powell ${ }^{1}$, Lance E. Courtney ${ }^{1,4}$, Maximilian H.-W. Schmidt ${ }^{2,3}$, Ari Feder ${ }^{1}$, Alexander Vogel $^{2}$, Yimin $\mathrm{Xu}^{1}$, David A. Lyon ${ }^{1}$, Kathryn Dumschott ${ }^{2}$, Marcus McHale ${ }^{5}$, Ronan Sulpice ${ }^{5}$, Kan Bao $^{1}$, Asha Duhan ${ }^{1}$, Asis Hallab ${ }^{3}$, Alisandra K. Denton ${ }^{2}$, Lukas A. Mueller ${ }^{1}$, Saleh Alseekh ${ }^{6,7}$, Jie Lie $^{8}$, Cathie Martin ${ }^{8}$, Alisdair R. Fernie ${ }^{6,7}$, Sarah R. Hind ${ }^{9}$, Gregory B. Martin ${ }^{1,10}$, Zhangjun Fei ${ }^{1}$, James J. Giovannoni ${ }^{1,11}$, Susan R. Strickler ${ }^{1^{*}}$, Björn Usadel ${ }^{2,3^{*}}$

${ }^{1}$ Boyce Thompson Institute, Ithaca, New York 14853

${ }^{2}$ Institute for Biology I, BioSC, RWTH Aachen University, 52474 Aachen, Germany

${ }^{3}$ IBG-4 Bioinformatics, Forschungszentrum Jülich, 52428 Jülich, Germany

${ }^{4}$ Plant Biology Section, School of Integrative Plant Sciences, Cornell University, Ithaca, NY 14853, U.S.A.

${ }^{5}$ Plant Systems Biology Lab, National University of Ireland, Galway, Ireland.

${ }^{6}$ Max-Planck-Institut für Molekulare Pflanzenphysiologie, Am Mühlenberg 1, 14476, Potsdam-Golm, Germany

${ }^{7}$ Center of Plant Systems Biology and Biotechnology, 4000 Plovdiv, Bulgaria

${ }^{8}$ Metabolic Biology Department, The John Innes Centre, Norwich Research Park, Norwich, NR4 $7 \mathrm{UH}, \mathrm{UK}$

${ }^{9}$ Department of Crop Sciences, University of Illinois at Urbana-Champaign, Urbana, IL, 61801, USA

${ }^{10}$ Plant Pathology and Plant-Microbe Biology Section, School of Integrative Plant Science, Cornell University, Ithaca, NY 14853, U.S.A.

${ }^{11}$ US Department of Agriculture-Agricultural Research Service, Robert W. Holley Center for Agriculture and Health, Ithaca, NY, USA.

*for correspondence srs57@cornell.edu and b.usadel@fz-juelich.de

\section{Summary}

Wild relatives of tomato are a valuable source of natural variation in tomato breeding, as many can be hybridized to the cultivated species (Solanum lycopersicum). Several, including Solanum lycopersicoides, have been crossed to $S$. lycopersicum for the development of ordered introgression lines (ILs). Despite the utility of these wild relatives and their associated ILs, limited finished genomes have been produced to aid genetic and genomic studies. We have generated a chromosome-scale genome assembly for Solanum lycopersicoides LA2951 using PacBio sequencing, Illumina, and $\mathrm{Hi}$-C. We identified 37,938 genes based on Illumina and Isoseq and compared gene function to the available cultivated tomato genome resources, in addition to mapping the boundaries of the S. lycopersicoides introgressions in a set of cv. VF36 $x$ LA2951 introgression lines (IL). The genome sequence and IL map will support the development of $S$. lycopersicoides as a model for studying fruit nutrient/quality, pathogen resistance, and environmental stress tolerance traits that we have identified in the IL population and are known to exist in S. lycopersicoides. 


\section{Introduction}

Tomato is the most widely consumed fruit crop with the greatest value world-wide. It is relatively rich in essential nutrients, particularly provitamin A, folate, vitamin $\mathrm{C}$, vitamin $\mathrm{E}$ and vitamin $\mathrm{K}$, iron, and calcium although these usually remain below their theoretical maximum levels because they are rarely targets for breeding. In addition, yield is often reduced significantly by losses caused by adverse environmental conditions, disease, pest damage, and post-harvest loss. The narrow germplasm base currently deployed in most breeding programs limits the potential for tomato improvement. Close wild relatives present opportunities to add enormous genetic diversity to tomato breeding programs and the means to identify and study genes that underpin useful novel variation. At least 14 wild relatives can be crossed to cultivated tomato, with varying degrees of difficulty, and have been used for decades in breeding programs (Grandillo et al., 2011). S. lycopersicoides, belongs to an outgroup to the tomato clade, and shows enhanced cold tolerance (Zhao et al., 2005), increased anthocyanin content (Rick et al. 1994), exceptional resistance to Botrytis cinerea (Guimarães, Chetelat and Stotz, 2004; Davis et al., 2009; Smith et al., 2014) and resistance to Pseudomonas (Mazo-Molina et al., 2019). While introgression lines (ILs) are available that can assist identification of alleles and mapping of loci conferring beneficial traits from S. Iycopersicoides (Canady, Meglic and Chetelat, 2005), few loci have been cloned partly due to a lack of a reference genome assembly for this species. Despite the importance of wild accessions in tomato breeding, only one such species, S. pennellii, has a reference quality genome (Bolger et al., 2014; Schmidt et al., 2017). Four species, S. pimpinellifolium (Tomato Genome Consortium, 2012; Razali et al., 2017), S. galapagense (Strickler et al., 2015), S. arcanum and S. habrochaites (The 100 Tomato Genome Sequencing Consortium et al., 2014) have draft de novo assemblies with small contig size and some have no gene annotation.

Of the biparental tomato IL populations developed to date, S. lycopersicoides LA2951 x . lycopersicum cV. VF36 ILs (Canady, Meglic and Chetelat, 2005) represents the widest cross. Due to the lack of a $S$. lycopersicoides genome, introgression boundaries of these lines are typically defined based on the published tomato reference genome (Tomato Genome Consortium, 2012). Genetic mapping resolution depends on these boundaries being well-defined and assumes lack of genome rearrangements or other modifications. Traditionally boundaries were defined by PCR markers dispersed across the genome, but more recent approaches increase precision by using SNPs derived from resequencing or RNA-Seq data (Gonda et al., 2019) (Chitwood et al., 2013). By basing introgression coordinates on the Heinz reference genome in wide crosses, information regarding differences in genome size is missing, which is relevant when comparing cultivated tomato to wild accessions that often have larger genome sizes. While many differences in genome size are accounted for by repetitive elements, gene gain and loss are also important to consider especially in regard to rapidly evolving genes involved in abiotic stress tolerances and resistance to biotic challenges. 
To improve understanding of the genes responsible for agriculturally important traits and increase the utility of wild species introgression lines, we generated a chromosome scale, reference genome and annotation for S. Iycopersicoides LA2951. We compared the genome to $S$. pennellii and $S$. lycopersicum to identify unique features that may be key to understanding the genetics of abiotic and biotic stress tolerance in S. lycopersicoides. Using RNA-seq, we mapped the introgressions of the ILs to both parental genomes to refine the genetic map of the population. The genome and associated map will facilitate improved understanding of the genes responsible for agriculturally important traits enabling their targeted introduction into tomato breeding lines.

\section{$\underline{\text { Results }}$}

\section{Genome assembly and feature prediction}

S. lycopersicoides LA2951 was selected for sequencing due to its traits of interest and the existence of introgression lines previously generated using this accession (Canady, Meglic and Chetelat, 2005). We assembled approximately 17 million PacBio reads with an average length of $6.29 \mathrm{kbp}$ totaling $107 \mathrm{Gbp}$ using two different genome assembly strategies relying on Canu (Koren et al., 2017) and Falcon (Chin et al., 2016). Canu produced an assembly with 17,507 contigs and an N50 value of 139,475 . This was the more contiguous assembly which captured a larger proportion of the BUSCO set (Waterhouse et al., 2017)(Supplemental table 1). Thus this assembly was selected for further scaffolding using Dovetail Chicago and $\mathrm{Hi}-\mathrm{C}$. The final assembly is $1.2 \mathrm{~Gb}$ in length (Table 1) and captures $97 \%$ of the BUSCO set (Supplemental figure 1 ). The genome assembly is larger than the $S$. lycopersicum assembly, which was expected based on kmer analysis (Table 1) and from previous observations (Rick et al., 1986). The final assembly $\mathrm{N} 50$ was $93.9 \mathrm{Mb}$ and $90 \%$ of the assembly was found in 12 pseudomolecules consistent with most of the assembly being captured by pseudochromosomes. Gaps within scaffolds ranged from 1 to 7,590 bp with a median of $970 \mathrm{bp}$. Approximately $1 \%$ of sites were found to be heterozygous, which is not surprising for a self incompatible species.

Figure 1 shows the twelve pseudochromosomes, along with repeat density and gene feature density. About $68 \%$ of the sequence contained in the pseudochromosomes was found to consist of repeats (Figure 2, Supplemental table 2). Genome annotation predicted a total of 37,938 putative genes (34,239 located on pseudomolecules) with a mean length of $4,388 \mathrm{bp}$ (Supplemental table 3). Genes had an average of 5.3 exons and a cds length of 1,232. We were able to identify $96 \%$ of the BUSCO set in the protein annotation (Supplemental figure 2).

Of all repeat classes, the long terminal repeat retrotransposons (LTR-RT) constituted the greatest proportion $(\sim 57 \%)$ of the $S$. lycopersicoides genome, as was the case with $S$. lycopersicum and S. pennellii genomes (Supplemental tables 2, 4, 5; Figure 2). For LTR-RT elements, Gypsy-type LTR-RTs were proportionately more abundant than Copia-type elements in each of the three genomes (Supplemental Tables 2, 4, 5). This result had been reported 
previously for S. lycopersicum and S. pennellii (Bolger et al., 2014). S. lycopersicoides also had a greater proportional abundance of younger LTR-RTs (with insertion times < 1MYA) compared to the other two genome assemblies (Supplemental figure 3 ).

\section{Functional annotation and gene families}

For annotation the automated functional annotation pipeline Mercator was used which reached classification and annotation rates in line with high quality genomes (Schwacke et al., 2019). The Mercator classification was used to investigate potential gene family expansion in the Solanum lycopersicoides genome assembly. This showed that in the case of the histone MLK kinase, there are six genes located on chromosomes 1, 8, 10, 11 and two copies on chromosome 12 both in S. pennellii and S.lycopersicum. However, in S. lycopersicoides there were three copies each in tandem configuration on chromosomes 1 and 8 (Supplemental table $\underline{6})$. In the case of protein elongation, there were multiple copies of genes encoding enzymes involved in synthesizing diphthamide, a modified histidine found only in elongation factor-2 (EEF2) including two DPH1 (Solyd06g065750, Solyd06g065840) and two DPH4 (Solyd12g069970, Solyd12g070080) diphthamide synthesis genes in S. lycopersicoides, respectively, but only one each in S. lycopersicum and $S$. pennellii. Similarly, there were twice as many deoxyhypusine synthase genes (contig2598g050010, Solyd01g058930, Solyd01g058950, Solyd02g069410). Deoxyhypusine synthase catalyzes the first step in conversion of a lysine residue in elF5A to the non-standard amino acid, hypusine using spermidine. Similarly, there were twice as many deoxyhypusine hydroxylase proteins (catalysing the second step in the synthesis of hypusine) (Solyd08g069360, Solyd08g069410) presumably generated by tandem duplication, encoded by the $S$. lycopersicoides genome compared to $S$. lycopersicum. This hypusine residue is essential for activity of elF5A.

Interestingly, within secondary metabolism, both the MVA and MEP pathways providing precursors for isoprenoid biosynthesis were expanded in S. lycopersicoides. The domesticated tomato and the $S$. pennellii genomes feature three HMG-CoA Synthases each, while the $S$. lycopersicoides genome features five (Solyd08g053200, Solyd08g072110, Solyd08g072130, Solyd08g072140, Solyd12g068050), due to a tandem expansion on chromosome 8. Furthermore, there might be more HMG-CoA reductase genes in S. lycopersicoides, but several were detected on unanchored contigs, only. Similarly, in the MEP pathway, in contrast to the domesticated tomato and $S$. pennellii, which feature one gene each encoding the D-xylulose 5-phosphate transporter, DXR 1-deoxy-D-xylulose 5-phosphate reductase, 4-diphosphocytidyl-2-C-methyl-D-erythritol kinase and 4-hydroxy-3-methylbut-2-enyl diphosphate reductase, $S$. lycopersicoides features two genes in each case.

We detected an expansion of some genes involved in flavonoid synthesis and modification. Whilst dihydroflavonol 4-reductase was triplicated (Solyd02g073780, Solyd02g073830, Solyd02g073850) compared to the domesticated tomato and $S$. pennellii, flavonol-3-O-glycoside-rhamnosyltransferase existed in two copies on chromosomes 3 and 5 in the genome of domesticated tomato, $S$. pennellii showed a tandem duplication of the gene on 
chromosome 3 and $S$. lycopersicoides showed a (near) tandem duplication on both chromosomes (Solyd03g076070, Solyd03g076130, Solyd05g056030, Solyd05g056040). Finally, we detected a putative expansion of the gene family encoding Phenylalanine Ammonia Lyase (PAL) in S. lycopersicoides.

In terms of co-enyzme metabolism, the genome featured several expansions in chlorophyll metabolism.

When investigating potential mechanisms for adaptations to an adverse environment, we noted that CAU1 encoding a histone methylase, which is an epigenetic suppressor of Ca signalling involved in stomatal closure, was duplicated in S. lycopersicoides, as well as an expansion of the salt overly sensitive pathway. Here, there was one SOS2 kinase which was duplicated in both wild tomato species compared to domesticated tomato.

In addition, the domesticated tomato as well as the $S$. pennellii genome harbors two SOS1 sodium:protein antiporters whereas there were four in $S$. lycopersicoides due to a tandem triplication on chromosome 4.

Related to immunity, we detected an expansion in S. lycopersicoides compared to the domesticated tomato genome and $S$. pennellii of genes encoding members of the $G$ protein family (eight versus five), and AGG1/2 G-gamma component (four versus three in S.pennellii or two in the domesticated genome) as well as for the LysM-RLK Lyk5 (two in S.lycopersicoides Solyd02g083440, Solyd02g083470). Related specifically to effector-triggered immunity, SGT1 exists in four copies (one being unplaced) but only two in the case of $S$. pennellii and domesticated tomato and there is a dramatic expansion of NLR receptor-encoding genes. Other genes encoding LysM-RLKs, which are typically involved in symbiosis or defense response, depending on species (Buendia et al., 2018), were expanded in S. lycopersicoides (Solyd02g078930, Solyd09g070440).

A total of 159,589 out of 208,760 proteins from Capsicum annum, S. tuberosum, $S$. lycopersicoides, S. pennellii, and S. lycopersicum were clustered into 25,763 orthogroups (Figure 3). There were 14,270 orthogroups containing 103,354 proteins that included at least one representative from each of the five species. A total of 29 gene families containing 185 genes were found to be unique to $S$. lycopersicoides. A total of 2,429 orthogroups containing 13,419 genes were unique to Solanum.

\section{Genome synteny and evolution}

Alignment and visualization of paired chromosomal segments of at least $8 \mathrm{~kb}$ and $92 \%$ sequence identity shows the high degrees of synteny between $S$. lycopersicoides and both $S$. lycopersicum and S. pennellii (Figure 4). Syntenic dot plots performed at a finer scale identified small inversions relative to the tomato genome (Supplemental figure 4). One inversion found on chromosome 10 is supported by a previously characterized inversion on chromosome 10 in $S$. 
lycopersicoides relative to S. lycopersicum and S. pennellii (Canady, Ji and Chetelat, 2006). Another inversion is found near the beginning of chromosome 4.

\section{Introgression mapping}

RNA-Seq libraries from 71 unique S. lycopersicum cv. VF36 x S. lycopersicoides LA2951 IL accessions were used to assemble genotype maps of the population (Sx-Sy). The map based on the current domestic parental reference genome (SL4.0) provides the coordinates delimiting the introgression boundaries within the reference parent background (Sx). An additional map based on the present $S$. lycopersicoides reference provides the coordinates which define the introgressed regions within the wild donor parent (Sy) (Figure 5). Approximately $87 \%$ of the $S$. lycopersicoides reference genome chromosomes is represented across these IL accessions. Our analysis revealed numerous previously unidentified introgressed segments and chromosomal features. For example, LA4245 is believed to harbor a single introgression on chromosome 4 (Canady, Meglic and Chetelat, 2005). Our results show that LA4245 harbors an additional $\sim 400 \mathrm{~kb}$ introgressed region at the distal end of the short arm of chromosome 4 . In addition, although this introgression appears on the end of the chromosome within LA4245, it is derived from a region closer to the centromere of chromosome 4 in S. lycopersicoides (Sy). This example exposes the risks of low resolution genotype mapping in missing introgressed segments and highlights the potential for error in downstream analyses. Without adequate genotyping, QTL mapping may be suspect. The present genome allowed for high resolution genotype mapping of associated ILs, providing a resource for the community to map QTLs more efficiently and to characterize the variation available from $S$. lycopersicoides.

\section{Discussion}

The last few years have seen a tremendous advance in plant genomic sciences driven by technologies such as long read PacBio and lately Oxford Nanopore sequencing (Bolger et al., 2019) as well as new technologies to obtain long range structural information such as optical mapping and Hi-C based information (Schreiber, Stein and Mascher, 2018). Indeed, it has been shown that these technological advances can be used to get (near) chromosome scale assemblies of sub-Gigabase genomes (Belser et al., 2018) and that they are particularly useful to unravel the genomes of wild species which are close relatives of important crops providing information about exotic germplasm and its use (Wu et al., 2018). However, whilst this would be particularly useful in the tomato clade, currently reference-style assemblies exist only for the domesticated tomato (Tomato Genome Consortium, 2012; Razali et al., 2017) and for the wild species Solanum pennellii (Bolger et al., 2014; Schmidt et al., 2017). Here, we present a novel high-quality chromosome-scale assembly for the genome of the wild tomato relative $S$. lycopersicoides, which represents an exotic germplasm donor, as together with its sister species S. sitiens it makes up the Solanum sect. Lycopersicoides, which is sister to the sect. Lycopersicon clade harboring the domesticated tomato (Knapp and Peralta, 2016). Unlike typical members of sect. Lycopersicon, S. lycopersicoides does produce large quantities of anthocyanins in the fruit, which might explain it harboring more 
flavonol-3-O-glycoside-rhamnosyltransferase and flavonol-3-O-glycoside-rhamnosyltransferase genes.

By making use of long read sequencing technology and Dovetail scaffolding, we provide not only a genome, where $>90 \%$ of the genome can be gathered in 12 chromosome-like scaffolds, but we also show very high completeness of the gene content. This is of particular importance as $S$. lycopersicoides has been used to establish an introgression line population (Canady, Meglic and Chetelat, 2005) which has been used to map several QTL for abiotic and biotic stress tolerance already, given the enhanced resilience of this wild species. Thus, this genome provides a unique reference to fine map and find causative genes underlying QTL when using this IL population. In line with previous marker based data, we also observed an inversion on chromosome 10 of the cultivated tomato relative to the ancestral $S$. lycopersicoides configuration (Pertuzé, Ji and Chetelat, 2002). We found an additional inversion between the cultivated tomato and $S$. lycopersicoides on chromosome 4 which had previously been speculated to be a hotspot as well (Albrecht and Chetelat, 2009).

In addition to improving QTL analyses, we also demonstrate the importance of this genome in assessing gene expression data. Given the large evolutionary distance between $S$. lycopersicoides and the domesticated tomato genome, simply mapping RNA-Seq data to the domesticated gene models showed a low mapping rate. Mapping to closely related relatives has been used (Hekman, Johnson and Kukekova, 2015) and was recommended as a better strategy than transcriptome assemblies (Vijay et al., 2013). Our data shows that this approach can be problematic, especially with distant crosses. While sophisticated bioinformatics pipelines allowing more flexible read alignment and iterative analysis of data and or normalization (Zhou et al., 2019) can reduce these problems, a high-quality reference genome provides the best solution. To improve gene expression analyses within ILs we propose a reference grafting strategy. Using the coordinates from the IL map and both parental reference genomes we assemble synthetic genomes specific for each IL (Supplemental Figure 5). These grafted reference genomes dramatically improve RNA-Seq mapping efficiency within introgressed regions of ILs. IL populations can be powerful tools for both breeding and research communities and the wild reference genome can improve their utility.

Finally, we show that our high-quality genome, together with the available tomato genome data, allows for the assessment of mechanisms of potential adaptation driven by, for example, tandem duplications leading to family expansion.

\section{Experimental Procedures}

\section{Plant Material}

LA2951 seeds were obtained from TGRC and transferred to soil and further cultivated in a greenhouse supplemented with artificial light to a light intensity of at least $200 \mu \mathrm{mol} \mathrm{m} \mathrm{m}^{-1}$ generated using Phillips hpi-t plus $400 \mathrm{w} / 645$ metal-halide lamps for $16 \mathrm{~h}$ a day. To preserve the 
genotype of the used cultivar, one plant was chosen and propagated by cuttings, which were used for DNA extraction.

RNA was prepared from fruit, flowers, and leaves by grinding tissue in liquid $\mathrm{N}_{2}$ into very fine powder, aliquoting $200 \mathrm{mg}$ of tissue into a $2 \mathrm{ml}$ tube, and extracting total RNA using Qiagen RNeasy mini kit. RNA-Seq libraries were prepared following the method described in (Zhong et al., 2011).

\section{Genome sequencing and assembly}

A single S. lycopersicoides LA2951 plant was chosen for all genome sequencing efforts. DNA was extracted from young leaves as previously described in (Bolger et al., 2014) and sequenced using PacBio P6C4 technology at Weill Cornell. In addition to this, one Illumina Nextera library, three Illumina TruSeq PCR-based and two Illumina PCR-free libraries were generated and sequenced on an Illumina MiSeq at Research Center Juelich following standard Illumina protocols. The PacBio sequence was assembled using Canu (main version 1.3, commit b147f45b114a9090568d78 fe409557bf5aeeb74f, with the parameter "genomeSize=1.3g") (Koren et al., 2017) and Falcon (Chin et al., 2016) assemblers (Table 1). BUSCO (Waterhouse et al., 2017) was used to assess the quality of the draft genome assemblies (Figure 2). The Canu genome was polished using Quiver with PacBio reads followed by three iterative rounds of Pilon (Walker et al., 2014) with Illumina paired-end reads and then scaffolded with SSPACE (Boetzer et al., 2011) and Illumina mate pair sequences (Table 1). The assembly was submitted to Dovetail Genomics for further scaffolding using Chicago and $\mathrm{Hi}-\mathrm{C}$ technologies. Scaffolding gaps were filled with PBJelly (English et al., 2012) using PacBio reads. Heterozygosity was estimated by mapping Illumina reads from genomics DNA to the final assembly with hisat2 and calling SNPs with the GATK (Poplin et al., 2018) pipeline.

\section{Annotation}

For gene model prediction, de novo repeats were predicted using RepeatModeler, known protein domains were removed from this set which was then used with RepeatMasker in conjunction with the Repbase library. For gene prediction, RNA was prepared from leaves, flowers, and fruits and sequenced on one lane of Illumina. Reads were mapped to the genome with hisat2 (Kim, Langmead and Salzberg, 2015). Portcullis (Mapleson et al., 2018) and Mikado (Venturini et al., 2018) were used to process the resulting bam files. PacBio IsoSeq data was also generated from a pool of leaves, fruits, and flowers and corrected using the Ice pipeline. Augustus and Snap were trained and implemented through the Maker pipeline, with Iso-Seq, proteins from Swiss-prot, and processed RNA-Seq added as evidence. Functional annotation and classification was performed using the automated Mercator pipeline (Schwacke et al., 2019).

\section{Repeat Analysis}


The genomes of S. lycopersicoides, S. lycopersicum, and S. pennellii were analyzed for LTR retrotransposons using LTRharvest (Ellinghaus, Kurtz and Willhoeft, 2008) with the parameters "-seqids yes -minlenltr 100 -maxlenltr 5000 -mindistltr 1000 -maxdistltr 20000 -similar 85 -mintsd 4 -overlaps best". The genomes were then analyzed using LTR_finder (Xu and Wang, 2007) with parameters "-D 20000 -d 1000 -L 5000 -I 100 -p 20 -C -M 0.85 -w 0". LTR_retriever was used to filter the LTR-RT candidates using default parameters, except that the neutral mutation rate was set at $1.0 \times 10^{-8}$ using the $-u$ parameter. This neutral mutation rate was selected as it has been used previously for tomatoes (Lin et al., 2014), assuming one generation per year (Beddows et al., 2017).

Candidate miniature inverted-repeat TEs (MITEs) were obtained using MITE-Hunter (Han and Wessler, 2010), with default parameters except for "-P 0.2". Output candidate MITEs were manually checked for their TSDs and TIRs as suggested in the MITE-Hunter manual. The candidate MITEs were also assigned to superfamilies based on best hits obtained by BLAST against the P-MITE database (http://pmite.hzau.edu.cn/download/), with an e-value cutoff of $1 \mathrm{e}^{-5}$. Any candidates that could not be unambiguously classified in this way were classified as unknowns.

The genomes were then masked using the repeat libraries generated by LTR_retriever and MITE-Hunter using Repeatmasker. Additional repeats were then identified de novo in the genomes using RepeatModeler. These repeats were classified using blastx against the Uniprot and Dfam libraries and protein-coding sequences were excluded using the script ProtExcluder.pl (Campbell et al., 2014). The masked genomes were then re-masked with Repeatmasker and the corresponding repeat libraries generated by RepeatModeler. Coverage percentages for the various repeat types were calculated using the fam_coverage.pl and fam_summary.pl scripts, both of which are included with LTR_retriever. All coverage percentages were calculated based on the length of the twelve chromosomes as the size for each genome.

\section{Genome Visualizations and Synteny}

Circular genome visualizations were generated using Circos (Krzywinski et al., 2009). For S. lycopersicoides, gene and repeat densities were calculated by generating $1 \mathrm{MB}$ windows and calculating percent coverage for each feature type (either annotated gene features or repeat elements identified in the repeat analysis) using a bedtools (Quinlan and Hall, 2010; Quinlan, 2014) command of the form: bedtools coverage -a [windows.bed] -b < ( sortBed -i [gene or repeat .bed file] ) > output.tab.

For synteny analysis, alignment of S. lycopersicoides to S. lycopersicum (SL4.0) and S. pennellii LA0716 v 2 (Bolger et al., 2014) was conducted with nucmer using the parameters --maxgap $=500$--mincluster=100, followed by delta-filter $-r$-q -i 92 -I 8000 and show-coords. Output was then used for links in Circos. 


\section{Gene family analysis}

For the Venn diagram, proteins from C. annuum v1.55 (Kim et al., 2014), S. tuberosum v3.4, S. lycopersicoides $\vee 1.0$, S. pennelli $\vee 2$ (Bolger et al., 2014), and S. lycopersicum Heinz $\vee 4.0$ (Tomato Genome Consortium, 2012) were clustered into orthogroups using OrthoFinder v 2.3.3 (Emms and Kelly, 2015).

\section{Introgression line maps}

RNA-Seq reads were first processed with Trimmomatic-0.36 (Bolger, Lohse and Usadel, 2014) to trim and filter low quality reads. Using Bowtie2 (Langmead and Salzberg, 2012) the reads were then aligned to databases of ribosomal RNA (Quast et al., 2013) and plant virus sequences (Zheng et al., 2017) to filter out non-mRNA reads. The reads were then used to call SNPs following the best practices in the Genome Analysis Toolkit documentation (McKenna et al., 2010). Both S. lycopersicum (SL4.0) and S. lycopersicoides reference genomes were used to establish a set of SNP markers within the population which distinguish between background and introgressed regions. Using a custom python script, SNPs that did not match either reference genome were filtered out. Only loci that were homozygous for a single parent or heterozygous with one base from each parent were used. This high density genotype marker map was then converted into a map of introgressions using SNPbinner (Gonda et al., 2019). Again, both parental reference genomes were used to map the introgression boundaries within the context of the domesticated background, as well as to positively define the introgressed segments within S. lycopersicoides.

\section{Accession Numbers}

The genome sequence is available at Solgenomics.net (Fernandez-Pozo et al., 2015) at https://solgenomics.net/organism/Solanum_lycopersicoides/genome

\section{Acknowledgements}

This work was supported by ERA-CAPS joint call 2 project Regulating Tomato quality through Expression (RegulaTomE) funded under grants DFG US98/7-1 (BU), FE552/29-1 (ARF), and BBSRC BB/N005023/1 (CRM), NSF IOS-1539831 and IOS-1855585 (JJG and ZF), NSF IOS-1546625 (GBM, SRS, and ZF), and the Triad Foundation (SRS, AF, SRH, and GBM). SA and ARF were additionally supported by funding from the Max-Planck-Society and the European Union project PlantaSyst (SGA-CSA No 664621 and No 739582 under FPA No. 664620). BU was supported by BMBF 031A536C. In addition, we want to thank Brian Bell for plant care, Surya Saha and Naama Menda for help with data handling and SGN synchronization and Roger Chetelat and the Tomato Genetics Resource Center for seeds.

\section{$\underline{\text { Contributions }}$}

$\mathrm{BU}$ and SRS managed the project. AFP, MHWS, ZF, JJG, SRS, and BU designed the project. 
AF, MHS, AV, KD, MM, AD, YX conducted DNA and RNA preparation and sequencing and plant experimental designs. AFP, LEC, MHS, DL, AD contributed new reagents and analytical tools. SA and ARF conducted metabolic analysis and interpretation. AFP, LEC, AKD, ZF, SRS, $\mathrm{BU}$, conducted the data analyses. BU, SRS, AFP, LEC wrote the manuscript with help from all authors.

Conflicts of Interest

Figures and tables 


\section{Figures}

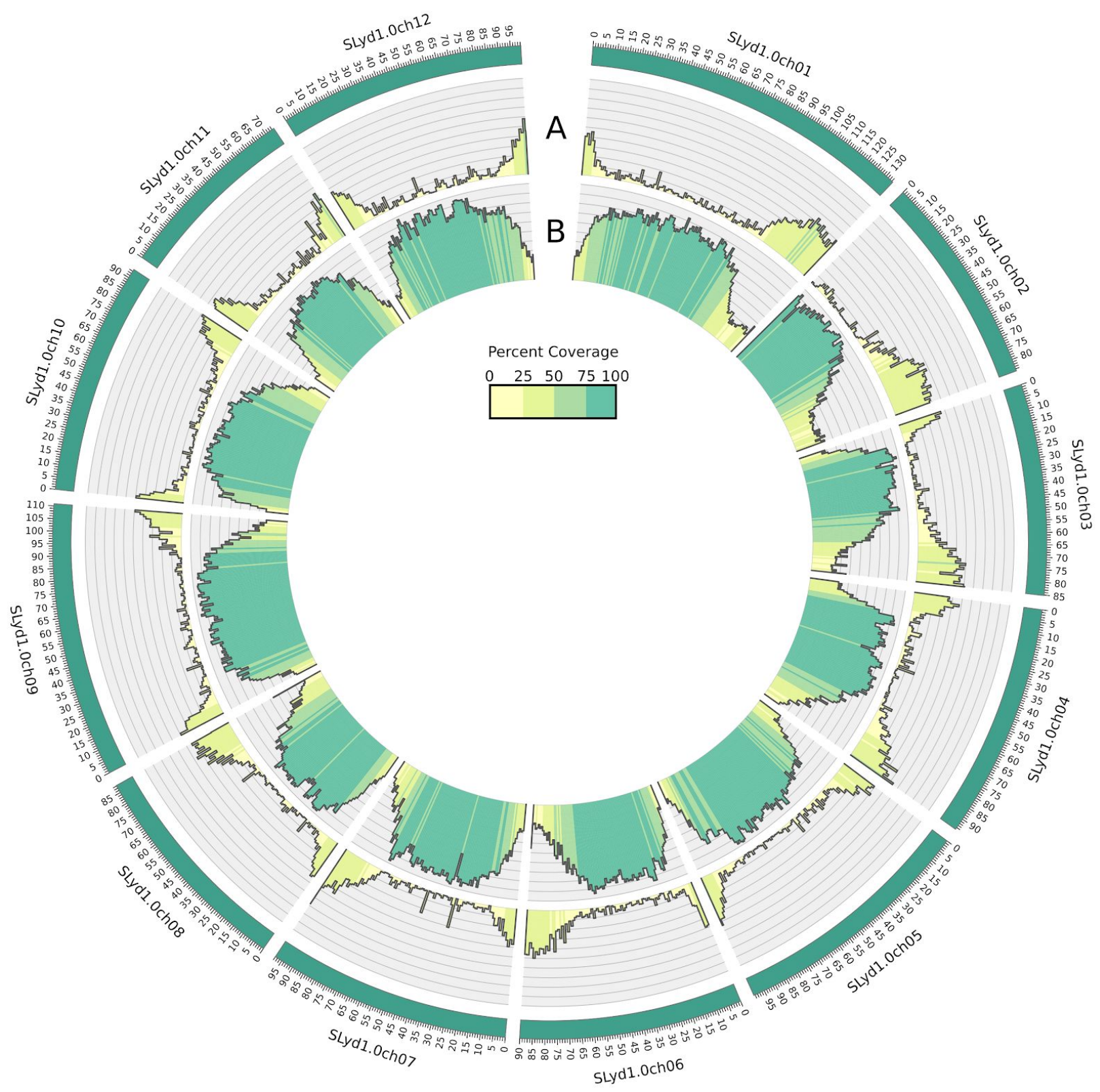

Figure 1 shows the 12 Solanum lycopersicoides chromosomes. Circos diagram illustrating the genome of $S$. lycopersicoides with (A) gene densities in the outer data track and $(B)$ repeat densities in the inner data track plotted relative to chromosome position. Densities are calculated for $1 \mathrm{Mb}$ windows and range from $0 \%$ to $100 \%$. Different colors are used for values falling within $0 \%$ to $25 \%, 25 \%$ to $50 \%, 50 \%$ to $75 \%$, and $75 \%$ to $100 \%$. 
A

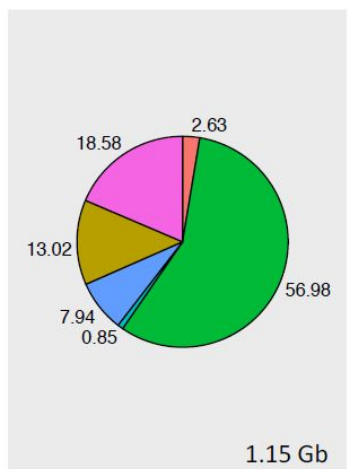

B

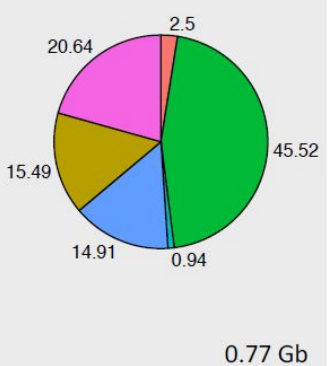

C

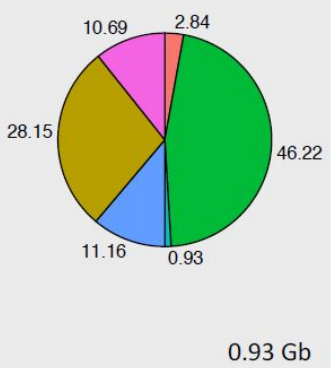

$0.93 \mathrm{~Gb}$

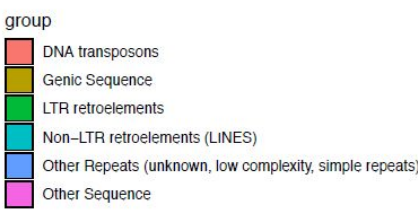

Figure 2 shows the Proportional contributions of component classes to the genomes of (A) $S$. lycopersicoides, (B) S. lycopersicum, and (C) S. pennellii. These figures relate to the 12 chromosomes for each genome, and the size of each 12-chromosome assembly is shown at the bottom of each panel.

\section{S. lycopersicoides}

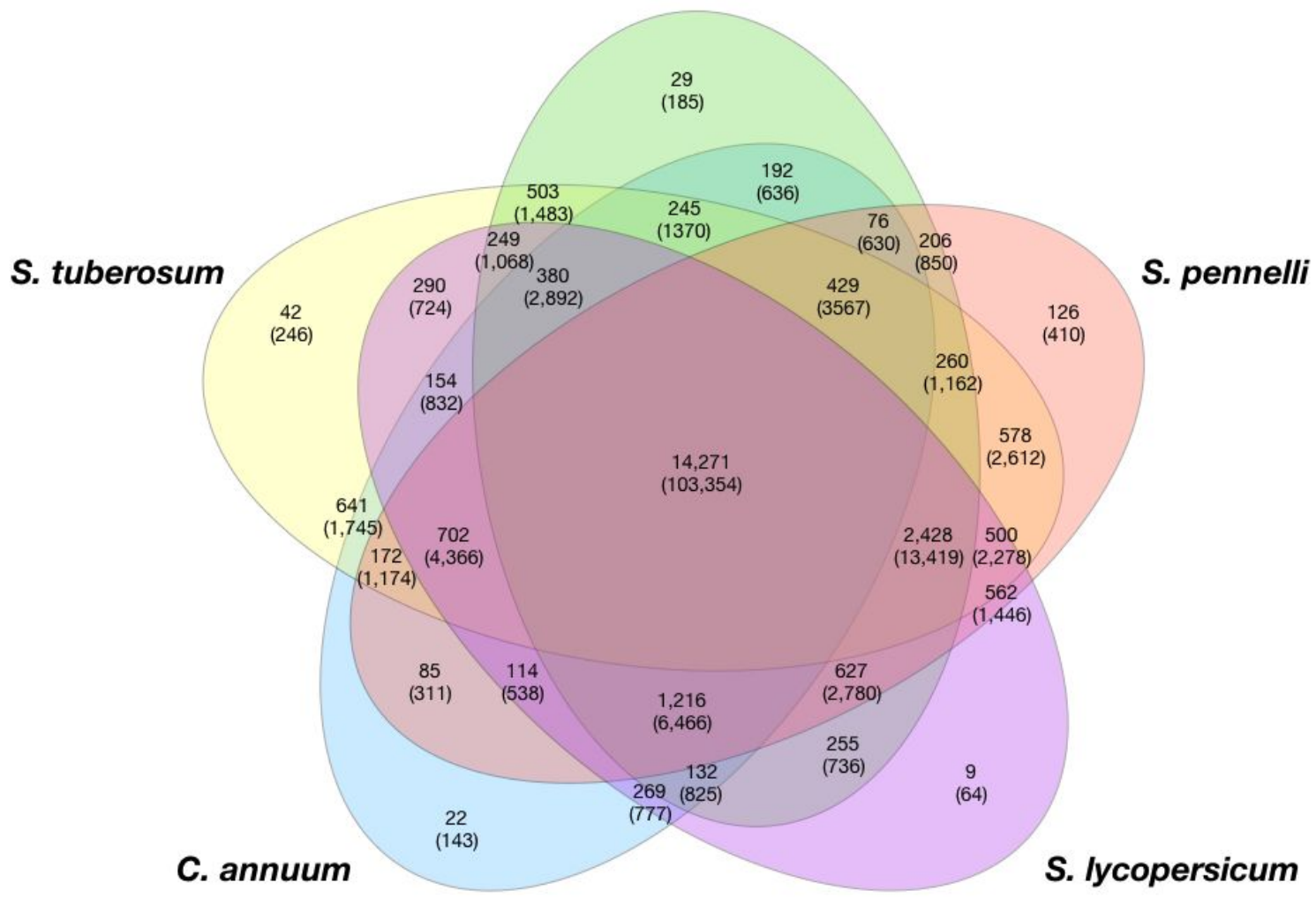


Figure 3 shows number of orthogroups overlapping amongst species. Values in brackets are the number of genes contained in the orthogroups.
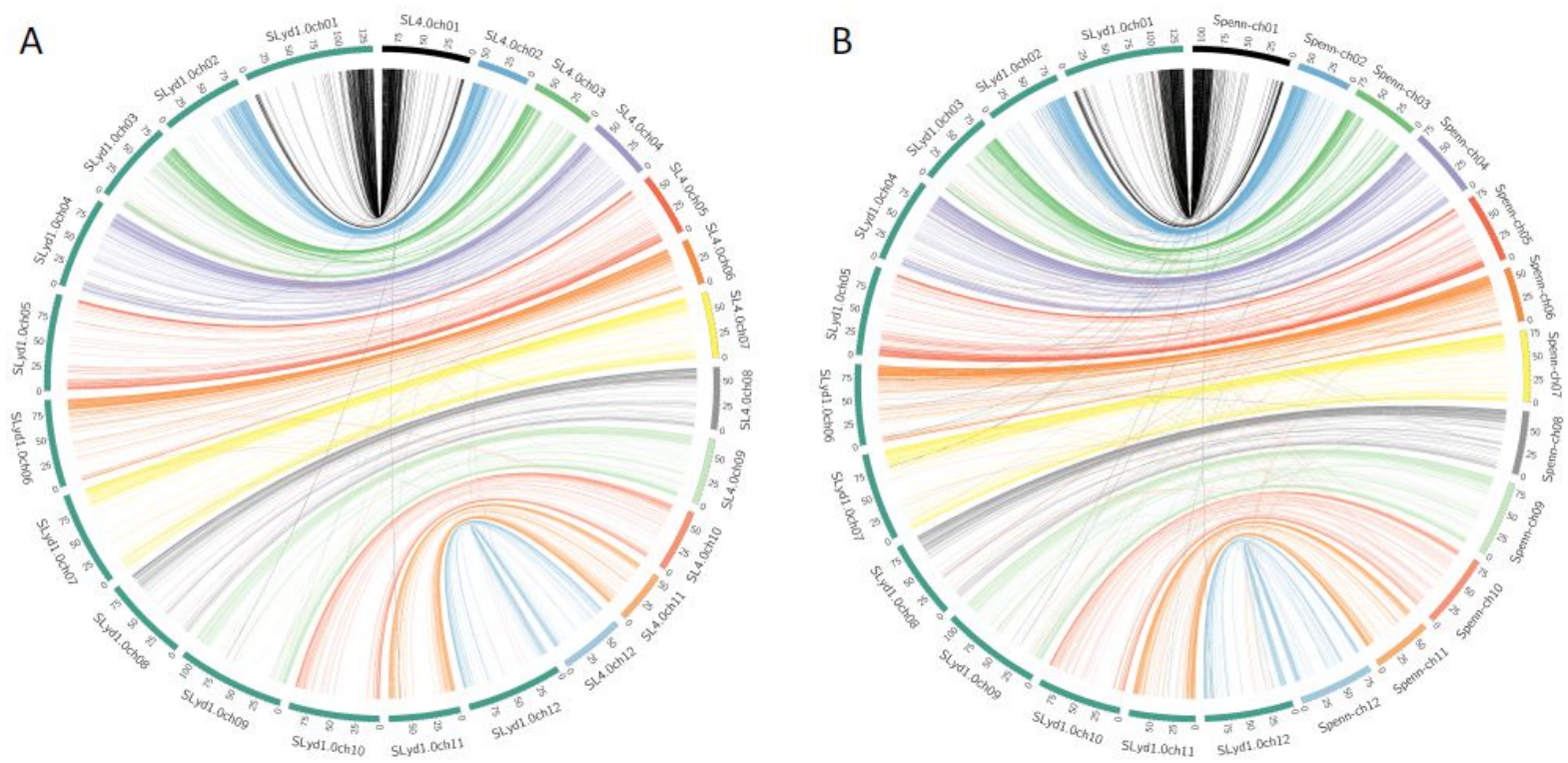

Figure 4 shows syntenic relationships (A) between S. lycopersicoides and S. lycopersicum as well (B) between $S$. lycopersicoides and $S$. pennellii. Lines in the center of the diagram link aligned sequences from one genome to the other. The color of the lines matches the color given to the corresponding S. lycopersicum or S. pennellii chromosome in the illustration. 
bioRxiv preprint doi: https://doi.org/10.1101/2020.04.16.039636; this version posted April 18, 2020. The copyright holder for this preprint (which was not certified by peer review) is the author/funder. This article is a US Government work. It is not subject to copyright under 17 USC 105 and is also made available for use under a CCO license.

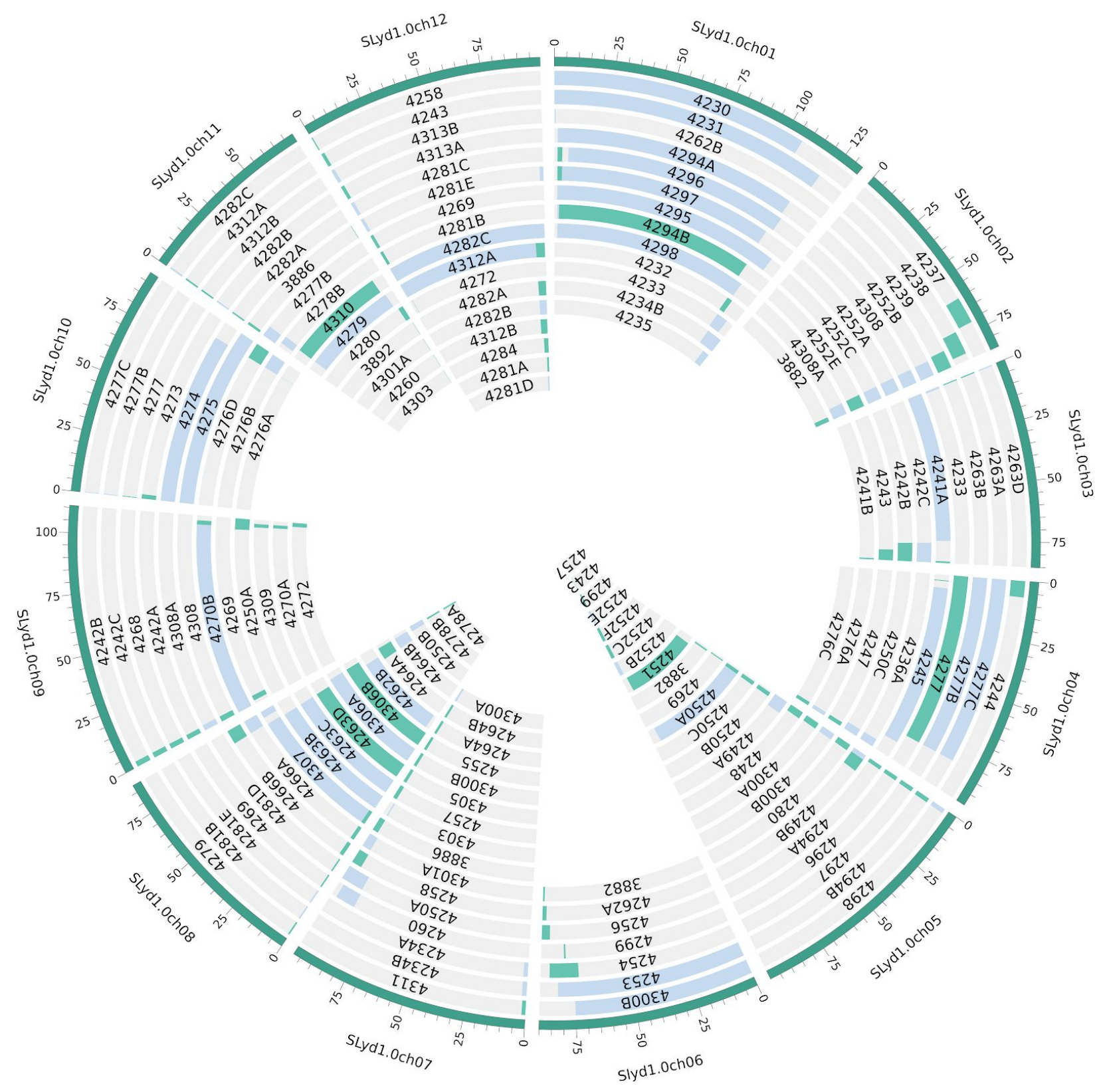

Figure $\mathbf{5}$ shows the mapping of $S$. lycopersicoides introgression lines onto the genome of $S$. lycopersicoides. Green indicates homozygous $S$. lycoperscoides mapping and blue indicates a mapping of heterozygous regions. 
bioRxiv preprint doi: https://doi.org/10.1101/2020.04.16.039636; this version posted April 18, 2020. The copyright holder for this preprint (which

was not certified by peer review) is the author/funder. This article is a US Government work. It is not subject to copyright under 17 USC 105 and is also made available for use under a CCO license.

Tables

Table 1 Summary statistics of $S$. lycopersicoides and other tomato genome assemblies

\begin{tabular}{|c|c|c|c|}
\hline & S. lycopersicoides & S. pennellii v2 & $\begin{array}{l}\text { S. lycopersicum } \\
\text { Heinz v } 4.0\end{array}$ \\
\hline No. of pseudomolecules & 12 & 12 & 12 \\
\hline longest sequence (Mbp) & 133.5 & 109.3 & 90.9 \\
\hline Contig N50 (bp) & 253,764 & 60,347 & $6,007,830$ \\
\hline total length (Mbp) & 1,152 & 926 & 782.5 \\
\hline $\begin{array}{l}\text { Total size (bp) of unanchored contigs } \\
\text { (\% of assembly) }\end{array}$ & $135,089,793(10.5)$ & $\begin{array}{r}63,101,713 \\
(6.4 \%)\end{array}$ & $9,643,250(1.2 \%)$ \\
\hline
\end{tabular}




\section{References}

Albrecht, E. and Chetelat, R. T. (2009) 'Comparative genetic linkage map of Solanum sect. Juglandifolia: evidence of chromosomal rearrangements and overall synteny with the tomatoes and related nightshades', TAG. Theoretical and applied genetics. Theoretische und angewandte Genetik, 118(5), pp. 831-847.

Aversano, R. et al. (2015) 'The Solanum commersonii Genome Sequence Provides Insights into Adaptation to Stress Conditions and Genome Evolution of Wild Potato Relatives', The Plant cell, 27(4), pp. 954-968.

Barchi, L. et al. (2019) 'A chromosome-anchored eggplant genome sequence reveals key events in Solanaceae evolution', Scientific reports, 9(1), p. 11769.

Beddows, I. et al. (2017) 'Population Genomics in Wild Tomatoes-The Interplay of Divergence and Admixture', Genome biology and evolution, 9(11), pp. 3023-3038.

Belser, C. et al. (2018) 'Chromosome-scale assemblies of plant genomes using nanopore long reads and optical maps', Nature plants, 4(11), pp. 879-887.

Boetzer, M. et al. (2011) 'Scaffolding pre-assembled contigs using SSPACE', Bioinformatics , 27(4), pp. 578-579.

Bolger, A. et al. (2014) 'The genome of the stress-tolerant wild tomato species Solanum pennellii', Nature genetics, 46(9), pp. 1034-1038.

Bolger, A. M. et al. (2019) 'Computational aspects underlying genome to phenome analysis in plants', The Plant journal: for cell and molecular biology, 97(1), pp. 182-198.

Bolger, A. M., Lohse, M. and Usadel, B. (2014) 'Trimmomatic: a flexible trimmer for Illumina sequence data', Bioinformatics , 30(15), pp. 2114-2120.

Buendia, L. et al. (2018) 'LysM Receptor-Like Kinase and LysM Receptor-Like Protein Families: An Update on Phylogeny and Functional Characterization', Frontiers in plant science, 9, p. 1531.

Campbell, M. S. et al. (2014) 'MAKER-P: a tool kit for the rapid creation, management, and quality control of plant genome annotations', Plant physiology, 164(2), pp. 513-524.

Canady, M. A., Ji, Y. and Chetelat, R. T. (2006) 'Homeologous recombination in Solanum lycopersicoides introgression lines of cultivated tomato', Genetics, 174(4), pp. 1775-1788.

Canady, M. A., Meglic, V. and Chetelat, R. T. (2005) 'A library of Solanum lycopersicoides introgression lines in cultivated tomato', Genome / National Research Council Canada = Genome / Conseil national de recherches Canada, 48(4), pp. 685-697.

Chin, C.-S. et al. (2016) 'Phased diploid genome assembly with single-molecule real-time sequencing', Nature methods, 13(12), pp. 1050-1054.

Chitwood, D. H. et al. (2013) 'A quantitative genetic basis for leaf morphology in a set of 
precisely defined tomato introgression lines', The Plant cell, 25(7), pp. 2465-2481.

Davis, J. et al. (2009) 'Mapping of loci from Solanum lycopersicoides conferring resistance or susceptibility to Botrytis cinerea in tomato', TAG. Theoretical and applied genetics. Theoretische und angewandte Genetik, 119(2), pp. 305-314.

Ellinghaus, D., Kurtz, S. and Willhoeft, U. (2008) 'LTRharvest, an efficient and flexible software for de novo detection of LTR retrotransposons', BMC bioinformatics, 9, p. 18.

Emms, D. M. and Kelly, S. (2015) 'OrthoFinder: solving fundamental biases in whole genome comparisons dramatically improves orthogroup inference accuracy', Genome biology, 16, p. 157.

English, A. C. et al. (2012) 'Mind the gap: upgrading genomes with Pacific Biosciences RS long-read sequencing technology', PloS one, 7(11), p. e47768.

Fantini, E. et al. (2013) 'Dissection of tomato lycopene biosynthesis through virus-induced gene silencing', Plant physiology, 163(2), pp. 986-998.

Fernandez-Pozo, N. et al. (2015) 'The Sol Genomics Network (SGN) -- from genotype to phenotype to breeding', Nucleic Acids Res, 43. D oi: 10.1093/nar/gku1195.

Giavalisco, P. et al. (2009) '13C isotope-labeled metabolomes allowing for improved compound annotation and relative quantification in liquid chromatography-mass spectrometry-based metabolomic research', Analytical chemistry, 81(15), pp. 6546-6551.

Gonda, I. et al. (2019) 'Sequencing-Based Bin Map Construction of a Tomato Mapping Population, Facilitating High-Resolution Quantitative Trait Loci Detection', The plant genome, 12(1). doi: 10.3835/plantgenome2018.02.0010.

Grandillo, S. et al. (2011) 'Solanum sect. Lycopersicon', in Kole, C. (ed.) Wild Crop Relatives: Genomic and Breeding Resources: Vegetables. Berlin, Heidelberg: Springer Berlin Heidelberg, pp. 129-215.

Guimarães, R. L., Chetelat, R. T. and Stotz, H. U. (2004) 'Resistance to Botrytis cinerea in Solanum lycopersicoides is Dominant in Hybrids with Tomato, and Involves induced Hyphal Death', European journal of plant pathology / European Foundation for Plant Pathology, 110(1), pp. 13-23.

Han, Y. and Wessler, S. R. (2010) 'MITE-Hunter: a program for discovering miniature inverted-repeat transposable elements from genomic sequences', Nucleic acids research, 38(22), p. e199.

Hekman, J. P., Johnson, J. L. and Kukekova, A. V. (2015) 'Transcriptome Analysis in Domesticated Species: Challenges and Strategies', Bioinformatics and biology insights, 9(Suppl 4), pp. 21-31.

Kim, D., Langmead, B. and Salzberg, S. L. (2015) 'HISAT: a fast spliced aligner with low memory requirements', Nature methods, 12(4), pp. 357-360.

Kim, S. et al. (2014) 'Genome sequence of the hot pepper provides insights into the evolution of 
pungency in Capsicum species', Nature genetics, 46(3), pp. 270-278.

Knapp, S. and Peralta, I. E. (2016) 'The Tomato (Solanum lycopersicum L., Solanaceae) and Its Botanical Relatives', in Causse, M. et al. (eds) The Tomato Genome. Berlin, Heidelberg: Springer Berlin Heidelberg, pp. 7-21.

Koren, S. et al. (2017) 'Canu: scalable and accurate long-read assembly via adaptive k-mer weighting and repeat separation', Genome research, 27(5), pp. 722-736.

Krzywinski, M. et al. (2009) 'Circos: an information aesthetic for comparative genomics', Genome research, 19(9), pp. 1639-1645.

Langmead, B. and Salzberg, S. L. (2012) 'Fast gapped-read alignment with Bowtie 2', Nature methods, 9(4), pp. 357-359.

Leisner, C. P. et al. (2018) 'Genome sequence of M6, a diploid inbred clone of the high-glycoalkaloid-producing tuber-bearing potato species Solanum chacoense, reveals residual heterozygosity', The Plant journal: for cell and molecular biology, 94(3), pp. 562-570.

Lisec, J. et al. (2006) 'Gas chromatography mass spectrometry-based metabolite profiling in plants', Nature protocols, 1(1), pp. 387-396.

Mapleson, D. et al. (2018) 'Efficient and accurate detection of splice junctions from RNA-seq with Portcullis', GigaScience, 7(12). doi: 10.1093/gigascience/giy131.

Mazo-Molina, C. et al. (2019) 'The Ptr1 locus of Solanum lycopersicoides confers resistance to race 1 strains of Pseudomonas syringae pv. tomato and to Ralstonia pseudosolanacearum by recognizing the type III effectors AvrRpt2/RipBN', Molecular plant-microbe interactions: MPMI. doi: 10.1094/MPMI-01-19-0018-R.

McKenna, A. et al. (2010) 'The Genome Analysis Toolkit: a MapReduce framework for analyzing next-generation DNA sequencing data', Genome research, 20(9), pp. 1297-1303.

Pertuzé, R. A., Ji, Y. and Chetelat, R. T. (2002) 'Comparative linkage map of the Solanum lycopersicoides and S. sitiens genomes and their differentiation from tomato', Genome / National Research Council Canada = Genome / Conseil national de recherches Canada, 45(6), pp. 1003-1012.

Poplin, R. et al. (2018) 'Scaling accurate genetic variant discovery to tens of thousands of samples', bioRxiv. doi: 10.1101/201178.

Potato Genome Sequencing Consortium et al. (2011) 'Genome sequence and analysis of the tuber crop potato', Nature, 475(7355), pp. 189-195.

Quast, C. et al. (2013) 'The SILVA ribosomal RNA gene database project: improved data processing and web-based tools', Nucleic acids research, 41(Database issue), pp. D590-6.

Quinlan, A. R. (2014) 'BEDTools: The Swiss-Army Tool for Genome Feature Analysis', Current protocols in bioinformatics / editoral board, Andreas D. Baxevanis ... [et al.], 47, pp. 11.12.1-34.

Quinlan, A. R. and Hall, I. M. (2010) ‘BEDTools: a flexible suite of utilities for comparing 
genomic features', Bioinformatics , 26(6), pp. 841-842.

Razali, R. et al. (2017) 'The genome sequence of the wild tomato Solanum pimpinellifolium provides insights into salinity tolerance', bioRxiv. doi: 10.1101/215517.

Rick, C. M. et al. (1986) 'Meiosis in sesquidiploid hybrids of Lycopersicon esculentum and Solanum lycopersicoides', Proceedings of the National Academy of Sciences of the United States of America, 83(11), pp. 3580-3583.

Rick, C. M. et al. (1994) 'Abg-a gene on chromosome 10 for purple fruit derived from S. lycopersicoides', Report of the Tomato Genetics Cooperative. Tomato Genetics Cooperative, 44, pp. 29-30.

Schmidt, M. H.-W. et al. (2017) 'De Novo Assembly of a New Solanum pennellii Accession Using Nanopore Sequencing', The Plant cell, 29(10), pp. 2336-2348.

Schreiber, M., Stein, N. and Mascher, M. (2018) 'Genomic approaches for studying crop evolution', Genome biology, 19(1), p. 140.

Schwacke, R. et al. (2019) 'MapMan4: A Refined Protein Classification and Annotation Framework Applicable to Multi-Omics Data Analysis', Molecular plant, 12(6), pp. 879-892.

Smith, J. E. et al. (2014) 'Resistance to Botrytis cinerea in Solanum lycopersicoides involves widespread transcriptional reprogramming', BMC genomics, 15, p. 334.

Strickler, S. R. et al. (2015) 'Comparative genomics and phylogenetic discordance of cultivated tomato and close wild relatives', PeerJ, 3, p. e793.

The 100 Tomato Genome Sequencing Consortium et al. (2014) 'Exploring genetic variation in the tomato ( Solanum section Lycopersicon ) clade by whole-genome sequencing', The Plant journal: for cell and molecular biology, 80(1), pp. 136-148.

Tomato Genome Consortium (2012) 'The tomato genome sequence provides insights into fleshy fruit evolution', Nature, 485(7400), pp. 635-641.

Venturini, L. et al. (2018) 'Leveraging multiple transcriptome assembly methods for improved gene structure annotation', GigaScience, 7(8). doi: 10.1093/gigascience/giy093.

Vijay, N. et al. (2013) 'Challenges and strategies in transcriptome assembly and differential gene expression quantification. A comprehensive in silico assessment of RNA-seq experiments', Molecular ecology, 22(3), pp. 620-634.

Walker, B. J. et al. (2014) 'Pilon: an integrated tool for comprehensive microbial variant detection and genome assembly improvement', PloS one, 9(11), p. e112963.

Waterhouse, R. M. et al. (2017) 'BUSCO applications from quality assessments to gene prediction and phylogenomics', Molecular biology and evolution. doi: 10.1093/molbev/msx319.

Wu, S. et al. (2018) 'Genome sequences of two diploid wild relatives of cultivated sweetpotato reveal targets for genetic improvement', Nature communications, 9(1), p. 4580. 
Xu, Z. and Wang, H. (2007) 'LTR_FINDER: an efficient tool for the prediction of full-length LTR retrotransposons', Nucleic acids research, 35(Web Server issue), pp. W265-8.

Zhao, L. et al. (2005) 'Investigation of Disease Resistance and Cold Tolerance of Solanum lycopersicoides for Tomato Improvement', HortScience: a publication of the American Society for Horticultural Science. American Society for Horticultural Science, 40(1), pp. 43-46.

Zheng, Y. et al. (2017) 'VirusDetect: An automated pipeline for efficient virus discovery using deep sequencing of small RNAs', Virology, 500, pp. 130-138.

Zhong, S. et al. (2011) 'High-throughput illumina strand-specific RNA sequencing library preparation', Cold Spring Harbor protocols, 2011(8), pp. 940-949.

Zhou, Y. et al. (2019) 'A statistical normalization method and differential expression analysis for RNA-seq data between different species', BMC bioinformatics, 20(1), p. 163. 